\title{
Dynamic Power-Loss Reduction for Over-Temperature Protection of Multilevel Class-D Amplifier
}

\author{
Bing-Shiun Hsieh and Chun-Wei Lin
}

\begin{abstract}
Class-D amplifier has been a popular device because of its high power efficiency. However, its ability of high power delivery also brings about over-temperature problem caused by consuming large power-loss over a long time. This paper therefore presents a control method to dynamically reduce the total power-loss of multilevel class-D amplifier according to the value of integrated temperature sensor. The driving (static) power-loss is reduced by limiting the driving current which can be achieved by reducing the output levels of the amplifier. In addition, the switching (dynamic) power-loss is diminished by segmenting the power transistors and gate drivers and selectively enabling parts of them according to the power demand. Therefore, the total power-loss of the amplifier is well controlled and reduced in order to prevent over-heating itself. Under the protection scheme, experimental results show that the total power-loss (excessive heat) and corresponding temperature difference on package can be reduced to only $15 \%$ of maximum value which demonstrates the effectiveness of proposed scheme.
\end{abstract}

Index Terms-Over-temperature protection, power-loss control, multilevel technique, class-D amplifier, PWM.

\section{INTRODUCTION}

High-power class-D audio amplifier is gradually popular among TV and home theatre sets because of its high efficiency [1]-[5]. However, its ability of high power delivery may also possibly over-heat itself because the power-loss of amplifier is increasing fast with the power demand, and therefore highlights the importance of system reliability. To prevent amplifier from damage, the over-current (OC), over-voltage $(\mathrm{OV})$ and over-temperature (OT) protection of high-power class-D amplifier (CDA) is in demand to reach the safe operating area (SOA) [6].

OC conditions are mainly arisen from loading variation or short circuit from output to power supply nodes, indeed, usually bring about the thermal runaway problem. This problem can be detected and lessened by restricting output current of amplifier within the acceptable limitation [6]-[9]. Moreover, OV is also called "clipping" because it happens when amplifier is over-driven. Under the OV situation, amplifier could be damaged because it is heated by maximum power-loss for a long time. The influence can be mitigated by using the automatic-gain-control (AGC) circuit to adjust the gain of pre-amplifier [10]-[14]. In addition, under normal

Manuscript received January 30, 2013; revised March 14, 2013.

B. S. Hsieh is with the Graduate School of Engineering Science and Technology, National Yunlin University of Science \& Technology (NYUST), Yunlin 640, Taiwan, R.O.C. (e-mail: g9610810@yuntech.edu.tw).

C. W. Lin is with the Department of Electronic Engineering, National Yunlin University of Science \& Technology (NYUST), Yunlin 640, Taiwan, R.O.C. (e-mail: linwei@yuntech.edu.tw). operation, class-D amplifier would be possibly over-heated because its power-loss is continuously increasing with output power demand. This large power-loss converts into excessive heat and raises the temperature on chip and PCB board, and hence seriously affects the system reliability. This problem is usually avoided by shutting amplifier down if the temperature on chip is larger than the maximum limits of packages. However, that will cause the audio holes problem [15]-[18]. In this work, we propose a dynamic power-loss reduction scheme to implement over-temperature protection for the multilevel class-D amplifier. Through dynamically controlling the total power-loss, the amplifier could continue operating well without shutting itself down when OT condition happens.

The paper is organized as follows. Section II introduces thermal resistance of packages which defines the relationship between the junction temperature and power-loss of chip. Section III describes design of multilevel class-D amplifier based on PWM and bridge-tied-load (BTL) configuration, and then presents design of proposed over-temperature protection which only operates when detecting OT condition. Finally, experimental results and conclusions are given in Section IV and Section V.

\section{Thermal Resistance OF ChIP PACKAGES}

Fig. 1 shows mechanisms of heat dissipation including conduction, convention and radiation. The relationship between the temperature of chip junction $T_{J}$ and ambient $T_{A}$ can be modeled by the two-resistor model which is further simplified as thermal resistance $\theta_{J A}$ of packages as shown in Fig. 1. This parameter $\theta_{J A}$ presents how the temperatures $T_{J}$ and $T_{A}$ and their difference relate to the power-loss $P_{\text {Loss }}$ and can be expressed as below:

$$
\theta_{J A}=\frac{T_{J}-T_{A}}{P_{\text {Loss }}}
$$

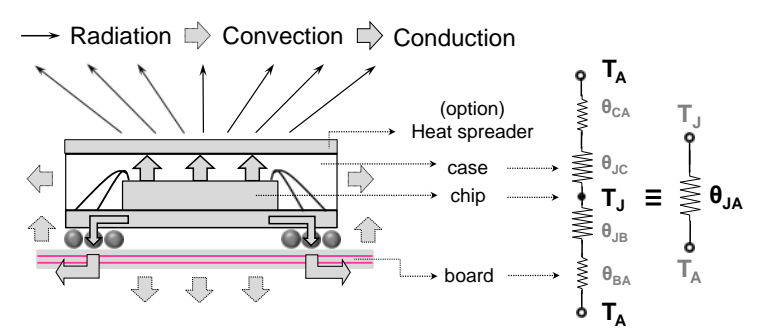

Fig. 1. Mechanisms of heat dissipation and thermal resistance of chip packages.

With fixed power-loss, the temperatures $T_{J}$ and $T_{A}$ and 
their difference $\Delta T$ are finally stabilized as shown in Fig. 2 and the thermal resistance $\theta_{J A}$ can be calculated by eq. (1). The most important thing is that, according to eq. (1), the temperature $T_{J}$ and $T_{A}$ and their difference $\Delta T$ on package can be lowered by reducing the power-loss $P_{\text {Loss }}$. In this work, OT protection will be implemented by using circuit design to reduce the power-loss instead of using complex package or extra device outside chip to enhance the heat dissipation, and hence reduces the product cost [19]-[20].

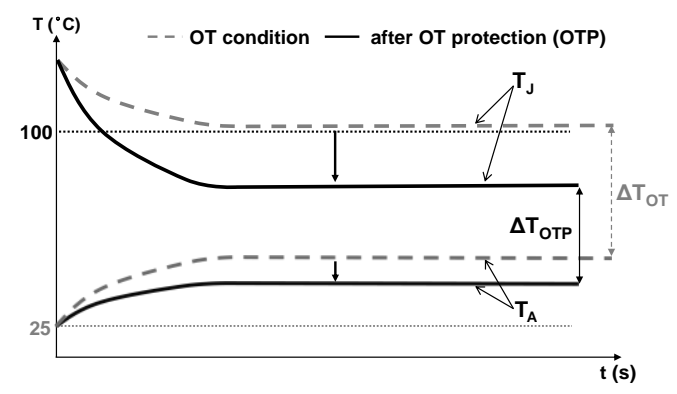

Fig. 2. Temperature transient of chip junction $T_{J}$ and ambient $T_{A}$ and their difference $\Delta T$ before and after OT protection.

\section{MultileVel Class-D AMPLIFIER With Proposed OVER-TEMPERATURE PROTECTION}

In this section, we present the multilevel class-D amplifier with proposed over-temperature protection shown in Fig. 3. The multilevel amplifier is based on the multilevel technique which consists of a time division adder (TDA) and a multilevel converter (MLC) [21]. Fig. 3 also shows that the power-loss is proportional to the output current directly because the amplifier can output signal as stairway, which simplifies the protection control. Moreover, the power-loss control loop uses an integrated temperature sensor to monitor chip condition and then the control logic produces corresponding control signals. Finally, the gain of pre-amplifier is adjusted to prevent OT condition. Therefore, the proposed method can enhance the reliability of the multilevel amplifier by dynamically controlling the power-loss and excessive heat. The relationship between the energy loss $W$ and corresponding excessive heat $H$ can be expressed as below:

$$
H=k \cdot\left(T_{J}-T_{A}\right) \propto W=P_{\text {Loss }} \cdot t=\left(P_{\text {Static }}+P_{\text {Dynamic }}\right) \cdot t
$$

where $k$ is heat coefficient of packages.

It is important to note that the power-loss $P_{\text {Loss }}$ comprises the static $P_{\text {Static }}$ and dynamic $P_{\text {Dynamic }}$ power-loss, therefore, the design of proposed protection scheme will focus on the reduction and control of their summation.

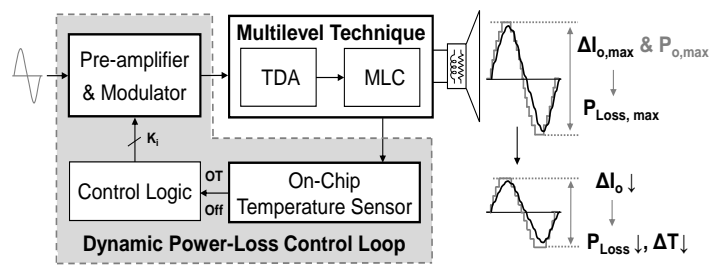

Fig. 3. Block diagram of the multilevel class-D amplifier with proposed over-temperature protection.

\section{A. The Multilevel Class-D amplifier}

Fig. 4 shows the implement of multilevel class-D amplifier which uses the multilevel technique to convert pulse width $D$ of PWM modulator $V_{P W M}$ into voltage levels $V$ of amplifier $V_{M L T}$ [21]. The relationship can be expressed as below:

$$
\begin{aligned}
V_{M L T}(V)=\Delta V & \cdot M \cdot D \propto V_{P W M}(D) \\
& =V_{S I G, \max } \cdot D \propto V_{S I G}(V)
\end{aligned}
$$

where $D, M$ and $\Delta V$ are duty ratio of PWM, voltage levels and voltage difference between adjacent levels.

According to the eq. (3), we can know that the multilevel technique is a linear conversion because the multilevel output $V_{M L T}$ represents the PWM output $V_{P W M}$ and audio signal $V_{S I G}$ as product of pulse width $D$, voltage levels $M$ and voltage difference $\Delta V$ between adjacent levels. The most important thing is that the multilevel technique simplifies the control of static power-loss reduction because it can output signal as stairway which makes the output levels proportionate to the output current and static power-loss. Moreover, the TDA output $V_{T D A}$ of the multilevel technique is a binary presentation of the multilevel output and hence helps for the control of dynamic power-loss reduction.

In this work, we firstly attempt to dynamically reduce the static power-loss for OT protection because the large output current of amplifier makes it dominate the total power-loss. This can be done by reducing the output levels of multilevel amplifier [21]-[23]. Then, we segment the power stage into several small power stages and use the TDA output to enable parts of them that are in need of conduction for reducing switching capacitance and dynamic power-loss. Thus, the total power-loss of the amplifier is well controlled and reduced in order to prevent over-heating itself under OT condition.

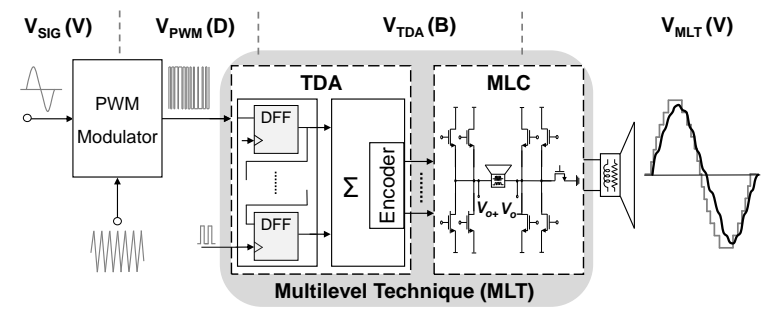

Fig. 4. Signals and operation of the multilevel class-D amplifier.

\section{B. The Static Power-Loss ( $\left.P_{\text {Static }}\right)$ Control}

As we known in eq. (1), thermal resistance of packages defines the relationship of temperatures $T_{J}$ and $T_{A}$ and their difference with power-loss, therefore, it is usually used to estimate the power-loss limitation of packages. With $85 \%$ efficiency of amplifier delivering $5 \mathrm{~V}$ sinusoidal signal to a $4 \Omega$ speaker, the power loss and corresponding temperature difference on package can be calculated as:

$$
\begin{gathered}
P_{\text {Loss }}=\left(\frac{5 / \sqrt{2}}{4}\right)^{2} \cdot 4 \cdot(1-0.85)=0.47 \mathrm{~W} \\
\Delta T=T_{J}-T_{A}=P_{\text {Loss }} \cdot \theta_{J A}=0.47 \cdot 65 \approx 30^{\circ} \mathrm{C}
\end{gathered}
$$

Eq. (5) means that PCB temperature will be larger than 
$70^{\circ} \mathrm{C}$ if chip temperature is over than $100^{\circ} \mathrm{C}$, which seriously degrades system reliability. For enhancing the reliability of amplifier, we attempt to reduce output current progressively for lowering the static power-loss and junction temperature as shown in Fig. 5. The static power-loss dominates power-loss when output current is heavy and is expressed as below:

$$
P_{\text {Static }}=I_{M L T, r m s}^{2} \cdot r_{\text {on }}
$$

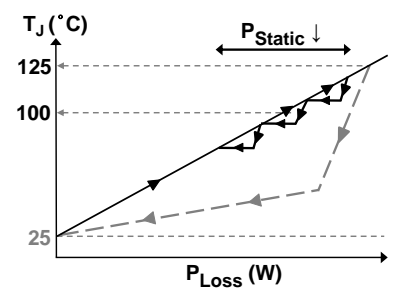

Fig. 5. Over-temperature protection according to the relationship between the chip junction temperatures $T_{J}$ and power loss $P_{\text {Loss }}$.

According to the linear relationship presented in eq. (3), the voltage levels (output current) of the multilevel amplifier can be reduced by limiting the variation of PWM pulses as shown in Fig. 6(a). This can be done by applying pre-amplifiers to audio or carrier input and adjusting their gain as shown in Fig. 6(b). In this work, we adjust the audio signal but not carrier because of the required high frequency and linearity of the carrier signal.

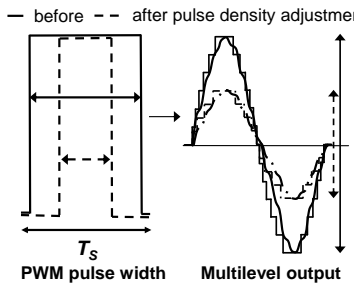

(a)

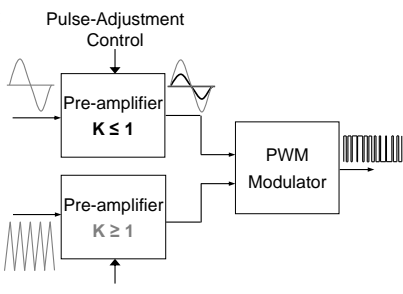

(b)
Fig. 6. (a) PWM pulses and output levels of the multilevel amplifier, and (b) the gain-adjustable pre-amplifier.

Fig. 7 presents transient response of voltage levels, powerloss and junction temperature of chip. During OT protection, the voltage levels of amplifier are progressively reduced to lower the power-loss and junction temperature. With a $65^{\circ} \mathrm{C} / \mathrm{W}$ thermal resistance of package, the proposed OT protection starts when the junction temperature is within $100 \sim 125^{\circ} \mathrm{C}$. In addition, the duration of protection time relies on PCB board and ambient condition for heat dissipation.

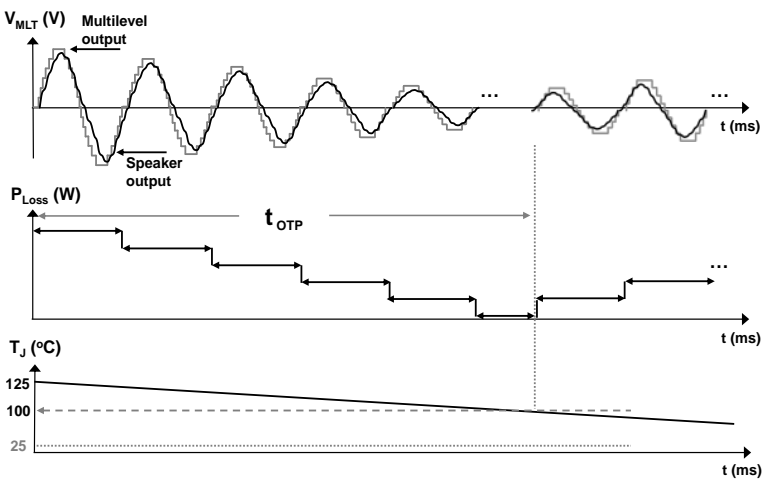

Fig. 7. Relationship between the reduction of voltage levels $V_{M T L}$, power-loss $P_{\text {Loss }}$ and chip junction temperature $T_{J}$.

\section{The Dynamic Power-Loss ( $\left.P_{\text {Dynamic }}\right)$ Control}

In addition to the control of static power-loss reduction, we attempt to further lower the dynamic power-loss for minimizing the total power-loss of multilevel class-D amplifier. Fig. 8 shows three sources and corresponding key factors of the total power-loss. The modulator \& multilevel generator and gate drivers consume only dynamic power-loss during switching transient because they drive capacitive load. On the contrary, the power transistors drive resistive load and mainly consume static power-loss. If we represent these power-loss respect to output power as shown in Fig. 9(a), only the static power-loss is raised because of increasing current delivery. Moreover, the static power-loss of power transistors and dynamic power-loss of gate drivers dominate the total power-loss in case of large and small power demand respectively. Thus, the critical factor of total power-loss minimization is how to reduce the dynamic power-loss $P_{S W, G D}$ of gate drivers with power demand as shown in Fig. 9(b). According to eq. (7), the corresponding region of high power efficiency after optimal control could be wider than before as shown in Fig. 9(c). With fixed supply voltage $V_{D D}$ and carrier frequency $f_{T r i}$, the dynamic power-loss of the gate drivers can be expressed as eq. (8) and lowered by reducing switching capacitance $C_{S W \text {,Total }}$ which depends on the aspect ratio of the power transistors.

$$
\begin{aligned}
& \eta(\%)=\frac{P_{\text {out }}}{P_{\text {out }}+P_{\text {Loss }}}=\frac{P_{\text {out }}}{P_{\text {out }}+\left[P_{\text {Dynamic }}+P_{\text {Static }}\right]} \\
& =\frac{P_{\text {out }}}{P_{\text {out }}+\left[\left(P_{S W, S I G}+P_{S W, G D}\right)+P_{\text {Static }}\right]} \\
& P_{S W, G D}=\frac{1}{2} V_{D D}^{2} \cdot f_{T r i} \cdot \sum_{i=1}^{9}\left[\left(T^{0}+T^{1}+\cdots+T^{N}\right) \cdot C_{P, i}\right] \\
& =\frac{1}{2} V_{D D}^{2} \cdot f_{T r i} \cdot \sum_{i=1}^{9} C_{S W, i}=\frac{1}{2} V_{D D}^{2} \cdot f_{T r i} \cdot C_{S W, \text { Total }}
\end{aligned}
$$

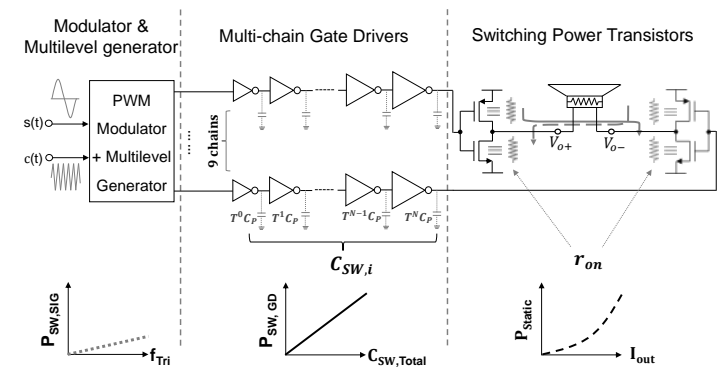

Fig. 8. Sources of power loss within multilevel class-D amplifier.

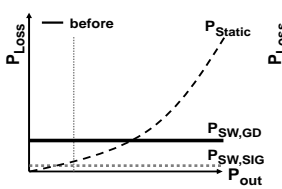

(a)

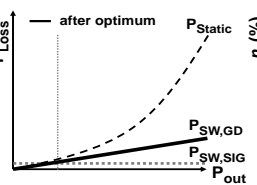

(c)
Fig. 9. Dynamic power-loss (a) before and (b) after optimum and (c) the corresponding power efficiency comparison

To reduce the switching capacitance $C_{S W \text {,Total }}$ for the reduction of the dynamic and total power-loss, we segment the power transistors and gate drivers into several small 
stages and then selectively enable parts of them that are in need of conduction as shown in Fig. 10. As we known in section III.A, the TDA output can be used for all digital control because it is a binary presentation of the multilevel output. For example, if the TDA output is $V_{T D A}=000001$, means a small power demand, we switch one of power stages to deliver current and disable the power PMOS and NMOS of the other five stages by setting their gate voltages to logic 1 and 0 . On the contrary, we switch all power transistors and their gate drivers for full power delivery if the TDA output is $V_{T D A}=111111$. Through applying proposed method, the segmented power stages are selectively enabled on demand that gives a substantial reduction in dynamic power-loss. Thus, the power efficiency of the amplifier is indeed improved over a wide range of output power demand, especially for small power delivery.

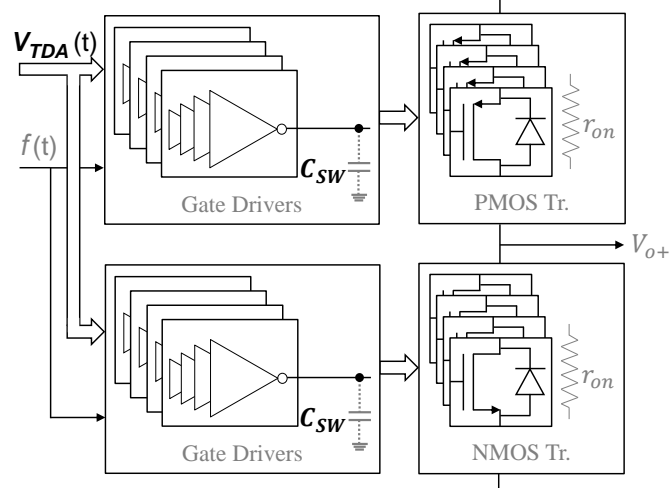

Fig. 10. Segmented power transistors and their gate drivers

\section{Integrated Temperature Sensor}

In this work, an integrated temperature sensor is used to identify over-temperature (OT) condition and enable proposed protection scheme. The temperature sensor uses a CTAT bandgap and a zero TC bandgap to output currents of $I_{C T A T}$ and $I_{Z T C}$ which are dependent on and independent of temperature respectively, two comparators are then used to point out two temperature points of $100^{\circ} \mathrm{C}$ and $125^{\circ} \mathrm{C}$ as shown in Fig. 11. The proposed scheme starts protection operation only if the chip junction temperature reaches the range of $100^{\circ} \mathrm{C} \sim 125^{\circ} \mathrm{C}$, but the multilevel amplifier will be shut down for safety if the junction temperature is still over than $125^{\circ} \mathrm{C}$ after the proposed OT protection shown in Fig. 5 and Fig. 9.
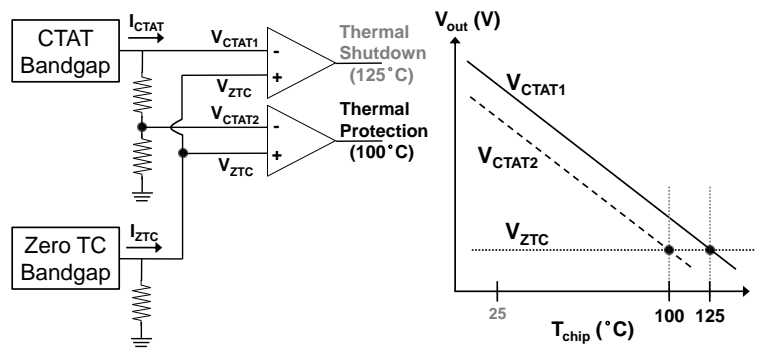

Fig. 11. Circuit scheme and transfer curve of integrated temperature sensor.

\section{EXPERIMENTAL RESULTS}

In this section, to demonstrate the improvement of system reliability, the multilevel class-D amplifier with proposed over-temperature protection shown in Fig. 3 was implemented by TSMC 5V-0.35um CMOS technology and directly drove a speaker with resistance $R_{L}=4 \Omega$ and inductance $L=33 \mu \mathrm{H}$. The amplifier outputs at most 13 levels of voltage signal and delivers $3.1 \mathrm{~W}$ of maximum power with $85 \%$ efficiency. Fig. 12 shows transient response of the multilevel amplifier which reduces the power-loss by lowering the voltage levels step by step when chip junction temperature $T_{J}$ is within $100^{\circ} \mathrm{C} \sim 125^{\circ} \mathrm{C}$ and therefore achieves the OT protection.

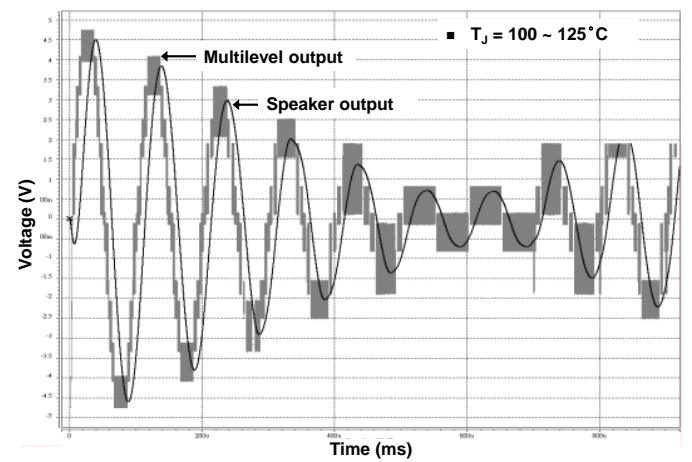

Fig. 12. Transient response of multilevel amplifier under OT protection.

With the amplifier and a $65^{\circ} \mathrm{C} / \mathrm{W}$ thermal resistance of package, the maximum power-loss $P_{\text {Loss }}=3.1 \mathrm{~W} \cdot(1-85 \%)$ $\doteq 0.47 \mathrm{~W}$ and corresponding temperature difference on package $\Delta T=0.47 \mathrm{~W} \cdot 65^{\circ} \mathrm{C} / \mathrm{W} \doteq 30^{\circ} \mathrm{C}$. This large temperature difference means that the amplifier may be possibly over-heated by itself because the package fails to dissipate excessive heat outside chip. In order to prevent the amplifier from damage, the total power-loss is reduced by limiting the output levels of the amplifier, which lowers the excessive heat and temperature difference. According to Fig. 13, the proposed method reduces the power-loss to $15 \%$ of maximum value when the output levels were reduced from 13 to 3 levels (reduced levels $=10$ ). Thus, the temperature difference is reduced to only $4.5^{\circ} \mathrm{C}$ which demonstrates the improvement of proposed scheme. Moreover, the power efficiency of the multilevel amplifier is well over $50 \%$ under OT protection as shown in Fig. 13.

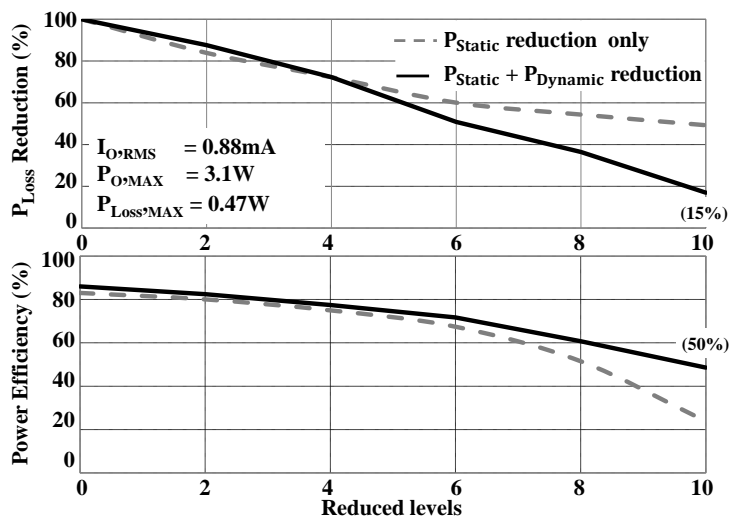

Fig. 13. Improvement of power-loss and power efficiency under OT protection.

\section{CONCLUSION}

This work has presented a control method to dynamically reduce the total power-loss and excessive heat for overtemperature protection and therefore enhances the reliability 
of the multilevel class-D amplifier. By applying proposed protection scheme to the amplifier, the output current and driving (static) power-loss can be decreased by reducing the voltage levels of the multilevel output step by step. Moreover, the switching (dynamic) capacitance and power-loss can be reduced by segmenting the power transistors and their gate drivers and using the TDA output to enable parts of them that is in need of conduction. Thus, the total power-loss of the amplifier is well controlled and reduced in order to prevent over-heating itself when OT condition happens. Without using complex packages or extra devices outside chip to enhance heat dissipation, the results of proposed method show that the total power-loss and excessive heat can be greatly reduced which demonstrates the improvement of proposed scheme.

\section{ACKNOWLEDGMENT}

The authors would like to thank the National Chip Implementation Center (CIC) of Taiwan for technical support and chip fabrication.

\section{REFERENCES}

[1] J. M. Liu, S. H. Chien, and T. H. Kuo, "A 100W 5.1-channel digital class-D audio amplifier with single-chip design," IEEE J. Solid-State Circ., vol. 47, no. 6, pp. 1344-1354, June 2012.

[2] M. Berkhout, "An integrated 200-W class-D audio amplifier," IEEE J. Solid-State Circ., vol. 38, no. 7, pp. 1198-1206, July 2003.

[3] M. Berkhout, "A 460W class-D output stage with adaptive gate drive," IEEE Int'l Solid-State Circ. Conf., Feb 2009, pp. 452-453.

[4] M. Paul and G. Eric, "A 20-W stereo class-D audio output power stage in 0.6- $\mu \mathrm{m}$ BCDMOS technology," IEEE J. Solid-State Circ., vol. 39, no. 11, pp. 1948-1958, Nov. 2004.

[5] B. Edoardo, M. Tiziana, and S. Fabrizio, "A high-efficiency 4x20W monolithic audio amplifier for automobile radios using a complementary D-MOS BCD technology," IEEE J. Solid-State Circ., vol. 31, no. 12, pp. 1895-1901, Dec. 1996.

[6] M. Berkhout, "Integrated overcurrent protection system for class-D audio power amplifiers," IEEE J. Solid-State Circ., vol. 40, no. 11, pp. 2237-2245, Nov. 2005.

[7] Y. Xu, F. Zhao, C. Hu, Y. L. Wu, and Y. Guan, "Self-recovering overcurrent protection circuit for class-D audio power amplifier," in Proc. IEEE Int'l Conf. on Microwave and Millimeter Wave Technol., May 2012, pp. 1-4.

[8] F. Cadete, D. Guilherme, J. Guilherme, and N. Horta, "Over-current detection circuit for integrated class-D amplifiers," in Proc. IEEE European Conf. on Circ. Theory and Design, Aug. 2011, pp. 29-31.

[9] J. J. Chen, Y. S. Hwang, and J. H. Su, "Integrated class-D amplifier with active current sensing suitable for alternating current switches," IEEE Trans. Ind. Electron., vol. 55, no. 8, pp. 3141-3149, Aug. 2008.

[10] M. F. Ross, "An investigation into how amplifier clipping is said to burn-out loudspeakers, and how limiters can save them," presented at the 89th Audio Eng. Soc. Convention, 1990, Preprint 2956.

[11] C. H. Nauta and H. E. Nordholt, "High-performance audio amplifier with AGC control and signal clipping for portable military radio," in Proc. IEEE European Solid-State Circ. Conf., Sept. 1986, pp. 89-91.

[12] J. Hauptmann, F. Dielacher, R. Steiner, C. C. Enz, and F. Krummenacher, "A low noise amplifier with automatic gain control and anticlipping control in CMOS technology," IEEE J. Solid-State Circ., vol. 27, no. 7, pp. 974-981, July 1992.

[13] Z. Y. Chang and D. Haspeslagh, "A CMOS differential buffer amplifier with accurate gain and clipping control," IEEE J. Solid-State Circ., vol. 30, no. 7, pp. 731-735, July 1995.

[14] J. Silva-Martinez and J. Salcedo-Suner, "A CMOS automatic gain control for hearing aid devices," in Proc. IEEE Int'l Symp. on Circ. and Syst. (ISCAS), May 1998, pp. 297-300.

[15] A. van Bezooijen, F. van Straten, R. Mahmoudi, and A. H. M. van Roermund, "Power amplifier protection by adaptive output power control," IEEE J. Solid-State Circ., vol. 42, no. 9, pp. 1834-1841, Sept. 2007.

[16] B. Krabbenborg, "Protection of audio amplifiers based on temperature measurements in power transistors," in Proc. IEEE Int'l Solid-State Circ. Conf., Feb 2004, pp. 374-375.

[17] A. van Bezooijen, F. van Straten, R. Mahmoudi, and A. H. M. van Roermund, "Over-temperature protection by adaptive output power control," in Proc. IEEE European Wireless Technol. Conf., Sept. 2006 , pp. $350-352$

[18] W. H. Dawes, "The design of a family of high-current switches with over-current and over-temperature protection," IEEE Trans. Instrum. Meas., vol. 47, no. 6, pp. 1492-1502, Dec. 1998.

[19] T. Y. Lee, "An investigation of thermal enhancement on flip chip plastic BGA packages using CFD tool," IEEE Trans. Compon. Packag. Technol., vol. 23, no. 3, pp. 481-489, Sept. 2000.

[20] B. Gogoi, R. Davies, J. Crowder et al., "New vertical silicon microwave power transistor structure and package with inherent thermal self-protection," in Proc. IEEE Int'l Microwave Symp., June 2009, pp. 569-572.

[21] C. W. Lin, B. S. Hsieh, and Y. C. Lin, "Enhanced design of filterless class-D audio amplifier," in Proc. IEEE Design, Automation \& Test in Europe, April 2009, pp. 1397-1402.

[22] C. W. Lin, B. S. Hsieh, and C. W. Chung, "PWM-based multilevel class-D amplifier with integrated over-current protection system," IEEE Conf. on Ind. Electr. and Appl., June 2010, pp. 1394-1398.

[23] C. W. Lin, B. S. Hsieh, "An anti-clipping protection system for multilevel class-D amplifier," in Proc. IEEE Int'l Conf. on Electron. Comput. and Telecommun and Information Technique, May 2011, pp. 129-132.

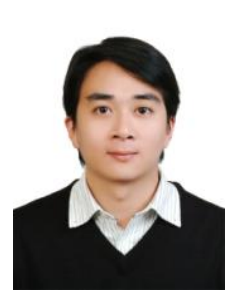

Bing-Shiun Hsieh received the B.S. and M.S. degree in electronic engineering from the National Yunlin University of Science and Technology (NYUST), Yunlin, Taiwan, R.O.C., in 2005 and 2007, respectively. He is currently working toward the Ph.D. degree at the Graduate School of Engineering Science and Technology in NYUST. His research interests are in the area of multilevel class-D audio amplifier, PWM power control, and mixed-signals integrated circuit

design.

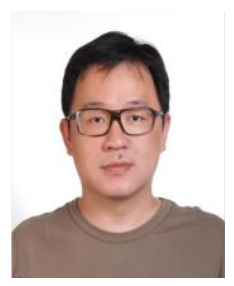

Chun-Wei Lin received B.S. degree in electronic engineering from Chung Yuan Christian University, Taiwan, R.O.C, in 1996. He obtained his Ph.D. Degree in Electronic Engineering, National Chiao Tung University, Taiwan, in 2002. From 2002 to 2006, he was with SPIROX Corporation, working in the area of design and test mixed signal circuit. In 2006, he joined the faculty of Electronic Engineering at National Yunlin University of Science and Technology in Taiwan. His research interests are in the area of integrated circuit design, VLSI testing, and computer aided design. He is a member of IEEE and IEICE. 